\title{
Speciation of Iron and Carbon in California Air Particulate Matter Using Complementary STEM/EELS and STXM Studies
}

\author{
VALERIE J. LEPPERT ${ }^{1 *}$, MATTHEW J. \\ ROBINSON $^{1}$, AJITH PATTAMMATTEL ${ }^{2}$, \\ PAUL ARONSTEIN ${ }^{2}$, PEGGY A. O'DAY ${ }^{2}$
}

${ }^{1}$ School of Engineering, University of California Merced, Merced, CA 95343, USA (correspondence: *vleppert@ucmerced.edu) ${ }^{2}$ Sierra Nevada Research Institute and School of Natural Sciences, University of California Merced, Merced, CA 95343, USA (poday@ucmerced.edu)

Identification of the physiochemical nature of particulate matter (PM) in ambient air is critical to unravel its biochemical responses and toxicity. Several individual components of natural and anthropogenic origin have been identified in PM, but the interaction between the individual components at a microscopic level is poorly known. Here, we have studied the speciation and association of iron $(\mathrm{Fe})$ and carbon, two significant components in particulate air pollution, using spatially resolved scanning transmission electron microscopy (STEM) equipped with electron energy-loss spectroscopy (EELS) and energy-dispersive X-ray spectroscopy (EDXS). The findings from STEM studies are supported with synchrotron-based scanning transmission X-ray microscopy (STXM) and spectroscopy. Ambient $\mathrm{PM}_{2.5}$ collected from Central and Southern California were found to contain submicron to nanoscale carbon nanoparticles (CNP) with adsorbed $\mathrm{Fe}$, identified from electron micrographs. Iron L-edge EELS and STXM data in comparison with reference compounds indicated that the adsorbed Fe on CNP is mostly in the 3+ oxidation state. Carbon K-edge X-ray absorption spectra showed that the carbon surface is oxidized to carboxylic acid groups. Moreover, both EELS and energy-filtered TEM (EFTEM) identified the presence of 20-30 nm Fe particles, with mixed $3+$ and $2+$ oxidation states, aggregated with CNPs, which may be the result of a combustion process. In $\mathrm{PM}_{2.5}$ from Central California, EELS showed nanoparticles with $\mathrm{Fe}(0)$ oxidation state and the presence of $\mathrm{Cr}$ and $\mathrm{Ni}$ in addition to $\mathrm{Fe}$, indicative of stainless steel. The specific chemical state of metal species in atmospheric nanoparticles is important to understand, as it may induce cellular inflammatory and antioxidant responses following inhalation. 